\title{
A Mysterious Case of Recurrent Acute Hyperammonemic Encephalopathy
}

\author{
Venkata Satish Pendela ${ }^{1}$, Pujitha Kudaravalli ${ }^{2}$, Anisleidys Munoz ${ }^{1}$, Gaby Razzouk ${ }^{1}$ \\ 1. Internal Medicine, Rochester General Hospital, Rochester, USA 2. Internal Medicine, State University of New York \\ (SUNY) Upstate Medical University, Syracuse, USA
}

Corresponding author: Venkata Satish Pendela, pvsatish.135@gmail.com

\begin{abstract}
Ammonia is a well-recognized neurotoxin. Awareness about hyperammonemia, in the absence of liver cirrhosis, may help in lifesaving, prompt diagnosis, and treatment. We present a case of a 53-year-old male who presented to the emergency department (ED) with altered mental status (AMS). He was unresponsive with occasional eye opening. Initial labs were normal except for mildly elevated blood alcohol level. Serum ammonia levels were very high ( $305 \mathrm{umol} / \mathrm{L})$. He improved with lactulose. He had similar admissions later on. Urine orotic acid levels were high confirming ornithine transcarbamylase (OTC) deficiency. Noncirrhotic hyperammonemia as a cause of AMS remains a diagnosis of exclusion requiring high index suspicion. Very few cases of late inborn errors of urea cycle disorders (UCDs) have been reported in the literature. Our case highlights the importance of early diagnosis of UCDs and that outcome can be excellent if treated aggressively. Once identified, adult-onset forms of the UCDs have a good prognosis-largely due to the initiation of preventative measures and earlier recognition of exacerbations.
\end{abstract}

Received 03/19/2020 Review began 03/25/2020 Review ended 03/29/2020 Published 03/31/2020

๑) Copyright 2020

Pendela et al. This is an open access article distributed under the terms of the Creative Commons Attribution License CC-BY 4.0., which permits unrestricted use, distribution, and reproduction in any medium, provided the original author and source are credited.
Categories: Emergency Medicine, Internal Medicine, Gastroenterology

Keywords: non-cirrhotic hyperammonemia, urea cycle disorders, altered mental status

\section{Introduction}

Confusion and altered mental status (AMS) are seen in $2 \%$ of the patients visiting the emergency department [1]. Ammonia is a well-recognized neurotoxin, mostly implicated in hepatic encephalopathy (HE) [2,3]. HE is one of the major causes of toxic metabolic encephalopathies which can result in acute emergency department (ED) visits. Hyperammonemia is related to severe liver disease in $90 \%$ cases, the remaining $10 \%$ is constituted by other conditions leading to disturbances in its excretion or production [2]. Awareness about hyperammonemia, in the absence of severe hepatic disease, may help in lifesaving, prompt diagnosis, and treatment.

\section{Case Presentation}

A 53-year-old African-American male was brought to the ED after his family members found him unresponsive. The family did not notice any alcohol, needles or empty pill bottles inside the house. His past medical history was significant for exercise controlled hypertension. He had no known allergies. On examination, he was unconscious with occasional spontaneous eye-opening and left gaze preference. Heart rate was 75 beats per minute, blood pressure $130 / 70 \mathrm{mmHg}$, temperature $99^{\circ} \mathrm{F}$, and respiratory rate 10 per minute. Neurological exam did not reveal neck rigidity or altered tone. Abdomen exam was benign.

Computed tomography (CT) of the head and CT angiogram of the neck did not reveal any acute abnormalities. Initial blood work revealed normal hemoglobin (14 g/dl), serum sodium (139 meq/L), arterial blood gas analysis, liver enzymes, and bilirubin. Serum ammonia level was elevated at 305 umol/L (Table 1). 


\section{Cureus}

Investigation

White cell counts

Serum sodium

Blood urea nitrogen

Urine drug toxicology

TSH

Serum salicylate

Serum Acetaminophen level

Blood alcohol

AST

ALT

Total bilirubin

Serum Ammonia

MRI brain

Ultrasound abdomen

CT abdomen

CT head and neck
Result

$5.5 \times 10^{9} / \mathrm{L}$

139meq/L

$6 \mathrm{mg} / \mathrm{dl}$

Negative

$0.56 \mathrm{ulU} / \mathrm{ml}$

$<3 \mathrm{mg} / \mathrm{dl}$

$<10 \mu \mathrm{g} / \mathrm{ml}$

$0.089 \mathrm{~g} / \mathrm{dl} \%$

$40 \mathrm{U} / \mathrm{L}$

$26 \mathrm{U} / \mathrm{L}$

$0.4 \mathrm{mg} / \mathrm{dl}$

305umol/L

Normal study

No evidence of liver cirrhosis

Normal liver contour

No acute abnormalities

\section{TABLE 1: Investigations}

TSH- thyroid stimulating hormone, AST- aspartate aminotransferase, ALT- alanine aminotransferase, MRI- magnetic resonance imaging, CTcomputed tomography

An electroencephalogram (EEG) revealed diffuse encephalopathy (Figure 1).

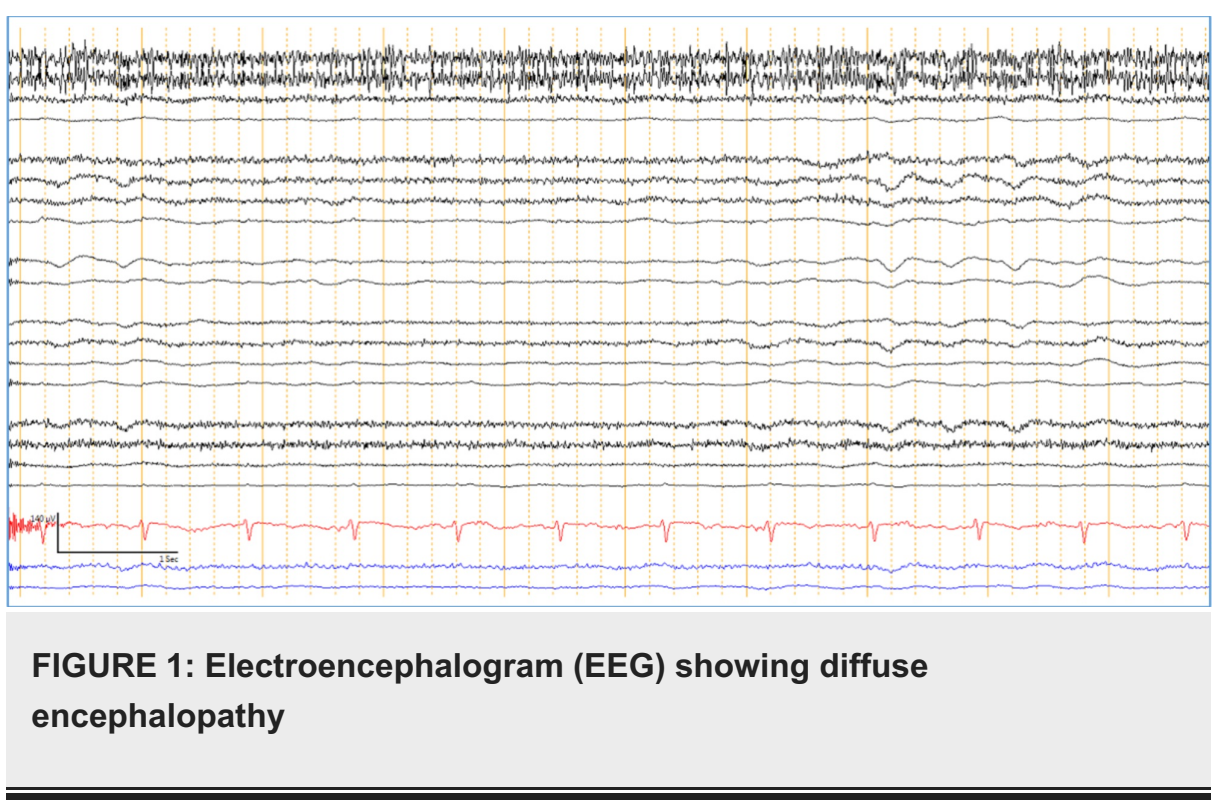

Magnetic resonance imaging (MRI) of the brain did not show any acute abnormality (Figure 2). 


\section{Cureus}

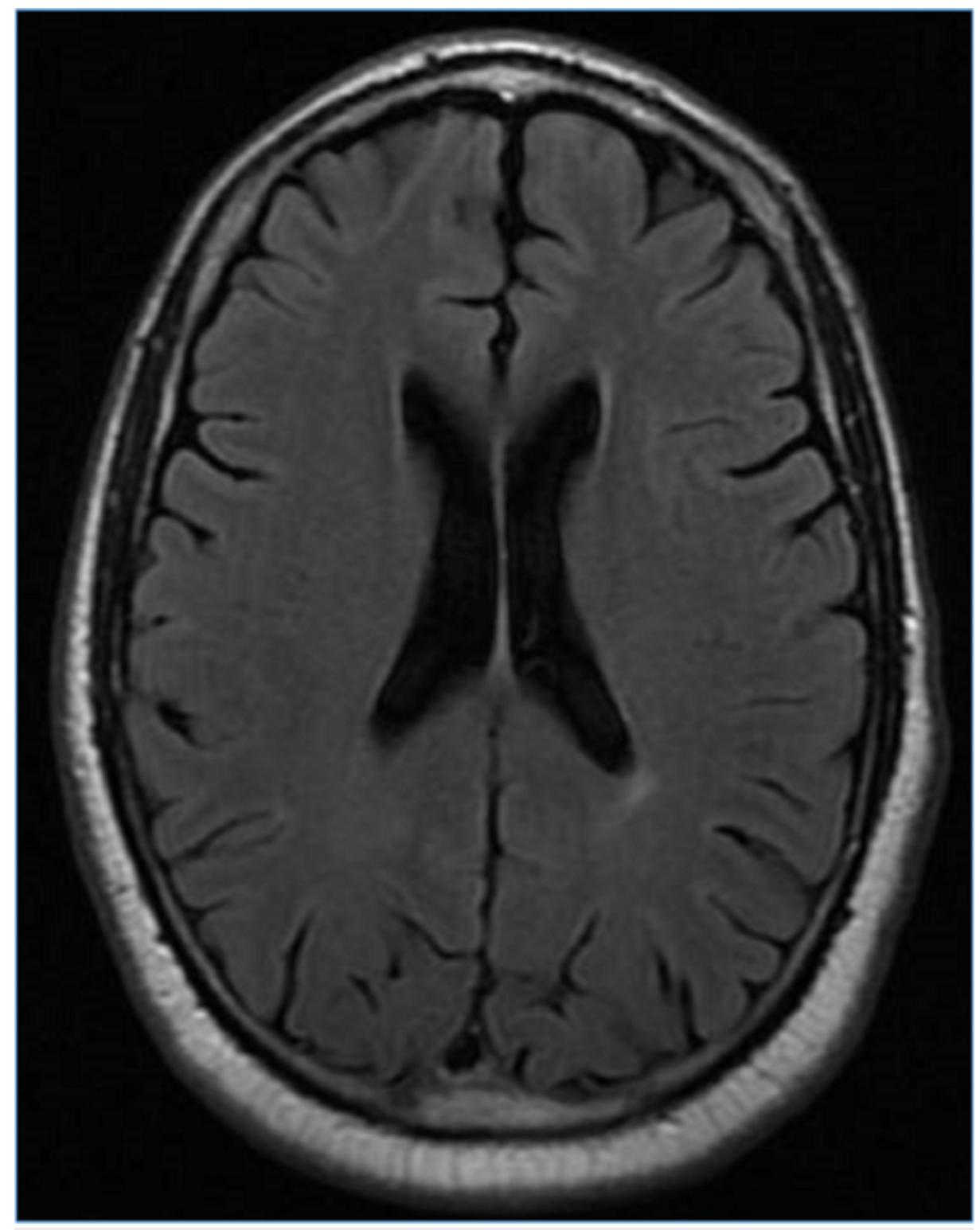

FIGURE 2: Magnetic resonance imaging (MRI) of the brain did not reveal any acute abnormality

A nasogastric tube was inserted and lactulose (30 g thrice daily) was administered. By the next day, he regained consciousness, and the serum ammonia decreased to $25 \mathrm{umol} / \mathrm{L}$. Ultrasound liver and CT abdomen ruled out cirrhosis. He was discharged with a prescription of lactulose.

He returned to the ED with another episode of unresponsiveness a month later and was found to have elevated serum ammonia of $213 \mathrm{umol} / \mathrm{L}$. He was not compliant with lactulose and he continued to have similar episodes of hyperammonemic encephalopathy. Serum amino acid levels were normal. Orotic acid in urine was high (>200 mmol/mol creatinine), clinching the diagnosis of ornithine transcarbamylase (OTC) deficiency. He was advised to consume a low protein diet and was prescribed L-carnitine along with lactulose.

\section{Discussion}

Ammonia directly affects neuronal electric activity by inhibiting the generation of both excitatory and inhibitory postsynaptic potentials. In a healthy human, the ammonia produced by protein digestion is converted into urea by the peri-portal hepatocytes, protecting the brain from the adversities of ammonia [4].

Secondary hyperammonemia occurs commonly in the presence of hepatic disorders leading to portosystemic shunting. Less common causes are described in Table 2. Primary hyperammonemia is a rare entity in adults and causes include milder forms of urea cycle disorders (UCD) like OTC, argininosuccinate lyase, 


\section{Cureus}

etc. (Figure 3) (Table 2) [5-11].

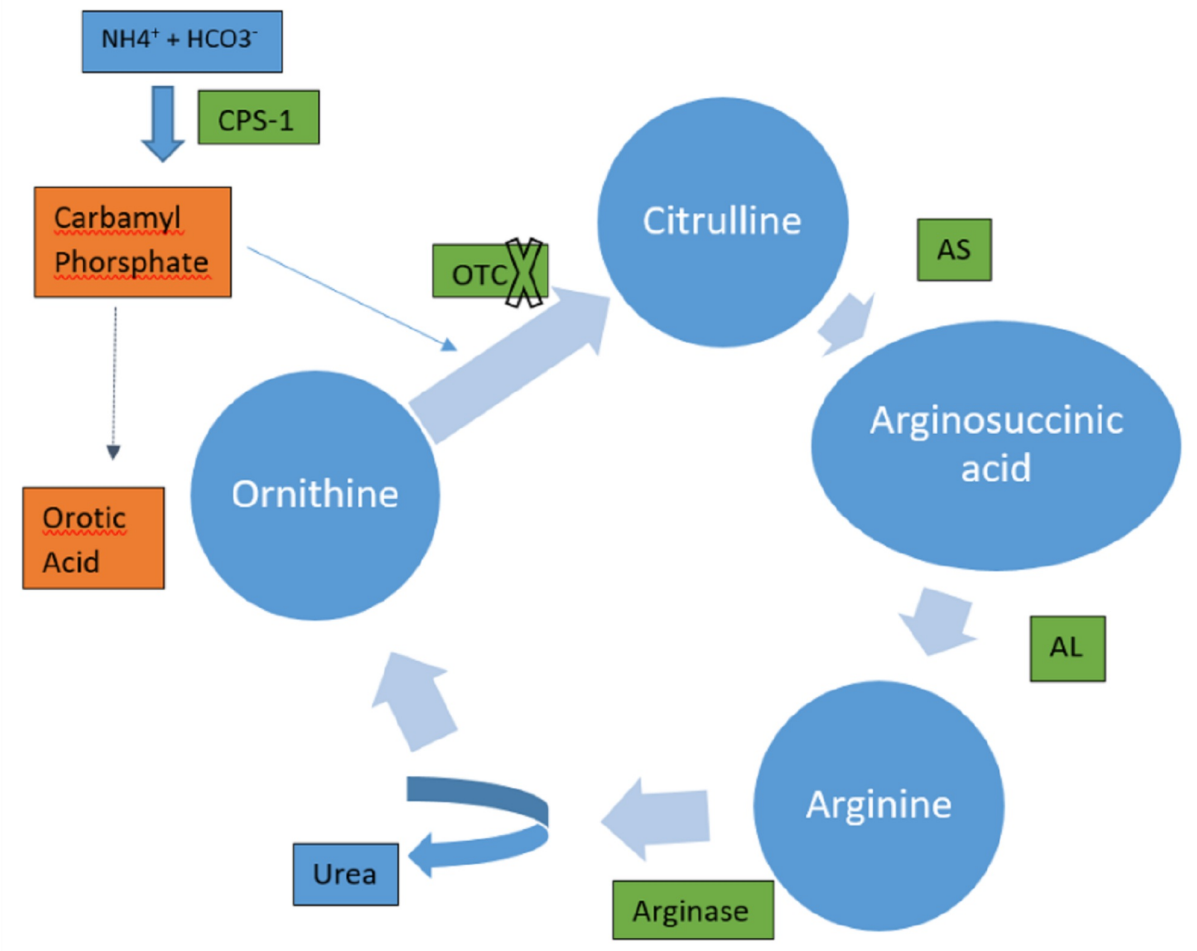

FIGURE 3: Urea cycle

OTC deficiency leads to increased orotic acid excretion.

AS- argininosuccinate synthetase, AL- Argininosuccinate lyase, CPS- carbamoyl phosphate synthetase I, OTC- ornithine transcarbamylase. 


\section{Cureus}

\section{Causes of non-hepatic hyperammonemia}

Urea cycle disorders (Primary Hyperammonemia) like Carbamoyl phosphate synthase I deficiency, Ornithine transcarbamoylase (OTC) deficiency etc.

\section{Secondary Hyperammonemia}

Portosystemic shunts

Urinary diversion- ureterosigmoidostomy with lleal conduit

Organic acidemias

Reye Syndrome

Drugs including Antiepileptics (valproate, carbamazepine), Anti-cancer drugs (5 fluorouracil)

Metabolic disorders like Fatty acid oxidation defects, Amino acid transport defects

Thyroid disease - Hashimoto's encephalopathy

Hematological disorders including Multiple myeloma and Acute myeloid leukemia

Post gastric bypass surgery

TABLE 2: Various causes of hyperammonemia without chronic liver disease

OTC deficiency is the most common inherited UCD. It is diagnosed by the high level of urinary orotic acid, secondary to the diversion of carbamoyl phosphate via the cytosolic pyrimidine synthetic pathway [12]. Even though it is more common in children (neonatal period), milder forms (homozygous males and heterozygous females) can present directly in adulthood $[13,14]$. Hyperammonemia can present as personality changes, sleep-wake cycle alterations, seizures, and confusion. Untreated acute severe hyperammonemia (ammonia levels >200 umol/L) can lead to comatose state as well as death [15]. This is due to severe cerebral edema and raised intracranial pressure. Cytotoxic and vasogenic oedema mechanisms have been implicated.

Isolated hyperammonemia without disturbances in other liver functions should prompt further investigations. A blood gas analysis showing acidosis points towards organic acidemias and respiratory alkalosis should raise suspicion of UCD. Classic magnetic resonance (MR) findings include the involvement of the insular cortex and gyrus for unknown reasons. As seen in our patient, MRI could be normal. Acute treatment is targeted towards ammonia lowering. Non-absorbable disaccharides (lactulose) help in decreasing ammonia production as well as absorption from the intestines [16]. Antibiotics like rifaximin and neomycin are approved for hepatic encephalopathy with hyperammonemia [17]. However, neomycin has serious adverse effects like ototoxicity, neurotoxicity, and nephrotoxicity limiting its use. Intra-cranial pressure lowering with mannitol or hypertonic saline is administered in patients with brain edema, seizures or coma. Hemodialysis is used in refractory hyperammonemia patients. Long-term dietary modifications with higher calorie to nitrogen ratio is preferred for UCDs. L-carnitine supplement helps in lowering the frequency of hyperammonemic attacks [18-20]. Liver transplant is an important treatment modality in patients with recurrent acute severe encephalopathy in UCDs.

The present work has been presented at the American College of Gastroenterology Conference 2019 [11].

(Abstract: Pendela VS, Munoz A, Razzouk G. A Mysterious Case of Non-Cirrhotic Recurrent Severe Hyperammonemia: What Urea-lly Need to Know. 2019 ACG Annual Meeting; October 2019).

\section{Conclusions}

Very few cases of late inborn errors of UCDs have been reported in the literature. The majority of these cases are fatal and present in previously healthy adults. Noncirrhotic hyperammonemia as a cause of AMS remains a diagnosis of exclusion and requires high index suspicion. Our case highlights the importance of early diagnosis of UCDs and that outcome can be excellent if treated aggressively. Once identified, adultonset forms of the UCDs have a good prognosis largely due to the initiation of preventative measures and earlier recognition of exacerbations.

\section{Additional Information \\ Disclosures}

Human subjects: Consent was obtained by all participants in this study. Conflicts of interest: In 
compliance with the ICMJE uniform disclosure form, all authors declare the following: Payment/services info: All authors have declared that no financial support was received from any organization for the submitted work. Financial relationships: All authors have declared that they have no financial relationships at present or within the previous three years with any organizations that might have an interest in the submitted work. Other relationships: All authors have declared that there are no other relationships or activities that could appear to have influenced the submitted work.

\section{References}

1. Hustey FM, Meldon SW: The prevalence and documentation of impaired mental status in elderly emergency department patients. Ann Emerg Med. 2002, 39:248-53.

2. Cichoz-Lach H, Michalak A: Current pathogenetic aspects of hepatic encephalopathy and noncirrhotic hyperammonemic encephalopathy. World J Gastroenterol. 2013, 18:26-34. 10.3748/wig.v19.11.26

3. Fraser CL, Arieff AI: Hepatic encephalopathy. N Engl J Med. 1985, 313:865. 10.1056/NEJM198510033131406

4. Norenberg MD, Rama Rao KV, Jayakumar AR: Signaling factors in the mechanism of ammonia neurotoxicity. Metab Brain Dis. 2009, 24:103-17. 10.1007/s11011-008-9113-6

5. Bijvoet GP, van der Sijs-Bos CJM, Wielders JPM, Groot OA: Fatal hyperammonaemia due to late-onset ornithine transcarbamylase deficiency. Neth J Med. 2016, 74:36-39.

6. Deutsch SI, Burket JA, Rosse RB: Valproate-induced hyperammonemic encephalopathy and normal liver functions: possible synergism with topiramate. Clin Neuropharmacol. 2009, 32:350-2. 10.1097/WNF.0b013e3181ac3615

7. Ah Mew N, Simpson KL, Gropman AL, Lanpher BC, Chapman KA, Summar ML: Urea Cycle Disorders Overview. GeneReviews ${ }^{\circledR}$, Seattle; 1993.

8. Adatia S, Poladia B, Joshi SR, Panikar V, Chauhan V, Hastak SM: Hyperammonemic coma presenting as Hashimoto's encephalopathy. J Assoc Physicians India. 2008, 56:989-91.

9. Rimar D, Kruzel-Davila E, Dori G, Baron E, Bitterman H: Hyperammonemic coma--barking up the wrong tree. J Gen Intern Med. 2007, 22:549-52.

10. Hawkes ND, Thomas GAO, Jurewicz A, Williams OM, Hillier CEM, McQueen INF, Shortland G: Non-hepatic hyperammonaemia: an important, potentially reversible cause of encephalopathy. Postgrad Med J. 2001, 77:717-722. 10.1136/pmj.77.913.717

11. Pendela VS, Munoz A, Razzouk G: A mysterious case of non-cirrhotic recurrent severe hyperammonemia: what urea-lly need to know. Am J Gastroenterol. 2019, 114:S1289.

12. Brusilow SW, Horwich AL: Urea cycle enzymes. The Metabolic and Molecular Basis of Inherited Disease 7th Edition. Scriven CR, Beaudet AL, Sly WS, Valle D (ed): McGraw-Hill, New York; 1995. 1187:221.

13. Rowe PC, Newman SL, Brusilow SW: Natural history of symptomatic partial ornithine transcarbamylase deficiency. N Engl J Med. 1986, 314:541-47. 10.1056/NEJM198602273140903

14. Batshaw ML, Roan Y, Jung AL, Rosenberg LA, Brusilow SW: Cerebral dysfunction in asymptomatic carriers of ornithine transcarbamylase deficiency. N Engl J Med. 1980, 302:482-5. 10.1056/NEJM198002283020902

15. Breningstall GN: Neurologic syndromes in hyperammonemic disorders . Pediatr Neurol. 1986, 2:253-262. 10.1016/0887-8994(86)90016-0

16. Als-Nielsen B, Gluud LL, Gluud C: Nonabsorbable disaccharides for hepatic encephalopathy. Cochrane Database Syst Rev. 2004, CD003044.

17. Bass NM, Mullen KD, Sanyal A, et al.: Rifaximin treatment in hepatic encephalopathy. N Engl J Med. 2010, 362:1071-1081. 10.1056/nejmoa0907893

18. Ohtani Y, Ohyanagi K, Yamamoto S, Matsuda I: Secondary carnitine deficiency in hyperammonemic attacks of ornithine transcarbamylase deficiency. J Pediatr. 1988, 112:409-414. 10.1016/S0022-3476(88)80321-4

19. Açikalin A, Dişel NR, Direk EÇ, Ilginel MT, Sebe A, Biçakçi Ş: A rare cause of postpartum coma: isolated hyperammonemia due to urea cycle disorder. Am J Emerg Med. 2016, 34:1324.e3-1324.e4. 10.1016/j.ajem.2015.12.013

20. Alameri M, Shakra M, Alsaadi T: Fatal coma in a young adult due to late-onset urea cycle deficiency presenting with a prolonged seizure: a case report. J Clin Med Case Rep. 2015, 9:267. 10.1186/s13256-015$0741-2$ 